\title{
Environmental and morphological dependence of the luminosity function of galaxies
}

\author{
M. J. Cuesta-Bolao and A. Serna \\ Departamento de Física y A.C., Universidad Miguel Hernández, 03202 Elche, Spain \\ Received 29 October 2002 / Accepted 31 March 2003

\begin{abstract}
In this paper we analyze the environmental and morphological dependence of the galaxy luminosity function (LF) by using a sample of homogeneous observational data: the Updated Zwicky Catalog (UZC). This sample is used to study the type-specific luminosity function of galaxies in the local field as well as in various environments which cover from isolated galaxies to moderately rich clusters.

We show that the overall LF cannot always be explained as a composition, taking into account the relative abundance of Hubble types, of type-dependent luminosity functions (LFT) with universal shapes. The environment, characterized by its richness $r$, also plays a crucial role in the shape of the specific LF for each Hubble type $T$. Nevertheless, the $r$ and $T$ dependence appears to be weak enough to allow for the definition of nearly universal luminosity functions, $\Phi(r, T)$ with coarse-grained bins on both $r$ and $T$.
\end{abstract}

Key words. galaxies: fundamental parameters

\section{Introduction}

The luminosity function (LF) of galaxies is central to many problems in extragalactic astronomy. For instance, the LF is presumably connected to the mass function of galaxies and, therefore, it could reflect both the characteristics of the primordial density fluctuation field and the astrophysical processes responsible for the formation and evolution of galaxies. Furthermore, the LFs for galaxies of individual Hubble types become more and more important for studies of the large-scale peculiar velocity field. It is needed to predict the redshift distribution of a galaxy type in various magnitude intervals which is essential to correct for the Malmquist bias in observational data (Jerjen \& Tamman 1997).

The galaxy LF has been the subject of numerous investigations (see the reviews by Felten 1977; Binggeli et al. 1988, and references therein). A considerable effort was first devoted to investigating the overall LF in clusters as well as in the field. In the pioneering works on this subject (e.g., Felten 1977), the shape of the LF appeared to be independent of the environment. However, the "universality" of the galaxy LF was soon discarded by an increasing number of works which found evidence of shape variations in LF as a function of the environment. The difference between cluster and field LFs has been interpreted as a result of the morphology-density relation of galaxies (Dressler 1980). According to this relation, high-density regions are dominated by elliptical and lenticular galaxies, whereas low-density regions are mainly populated

Send offprint requests to: A. Serna, e-mail: arturo.serna@umh. es by spiral and irregular galaxies. Each Hubble type has its specific luminosity function (LFT), and the overall LF is the sum of these LFTs. Consequently, it must be expected environmental effects on the overall LF because the morphological composition varies from cluster to cluster and with respect to the intercluster field.

The question was then centered on the possible universality of each specific LFT. From the study of galaxies in the local field, in small groups and in poor clusters, Binggeli et al. (1988) suggested that the luminosity function of each Hubble type could be universal. Different authors (e.g., Andreon et al. 1997; Jerjen \& Tamman 1997; Andreon 1998) supported such a suggestion by comparing LFTs measured in different environments. They in fact found that the shape of the LF of each wide galaxy type (Es, S0s, Ss) appears to be independent of the environmental density and, therefore, it could be expressed as a function $\Phi(T)$ which only depends on the Hubble type $T$. However, such a conclusion is controversial. Other authors (e.g., Valotto et al. 1997; Bromley et al. 1998; Ramella et al. 1999; Marinoni et al. 1999) found instead significant differences in LFTs of various environments and claimed that, in general, they must be expressed as a function $\Phi(\rho, T)$ of both density and morphology.

The possible universality of the LFTs still remains an open question, the answer of which could be important to understand the whole process of galaxy formation. As a matter of fact, recent works (e.g., Sommer-Larsen \& Dolgov 2001; Serna \& Domínguez-Tenreiro 2001; Sáiz et al. 2001; Sáiz et al. 2002) have succeeded in performing full consistent 
cosmological simulations where galaxies of different Hubble types are obtained. The individual properties of these objects (rotation curves, density profiles, ...) are close to those observed in the universe. A powerful test which could favor certain cosmological scenarios against others could be their implications on the shape of the LFTs.

The aim of this paper is to analyze both the environmental and morphological dependence of the galaxy luminosity function by using a sample of homogeneous observational data: the Updated Zwicky Catalog. This catalog will be used to study the type-specific LFs in different environments covering from isolated and field galaxies to galaxies in poor and moderately rich clusters. Special attention will be paid to control the main sources of uncertainties by means of a Montecarlo procedure.

This paper is organized as follows. In Sect. 2, we describe the different methods and techniques used throughout this paper as well as our criteria for selecting galaxy subsamples from the Updated Zwicky Catalog. In Sects. 3 and 4 we compute the LFTs for field and group galaxies, respectively. The question of the universality of the LFTs is then addressed in Sect. 5. Our results are compared to other works in Sect. 6 and, finally, our main conclusions are summarized in Sect. 7.

\section{Methodology}

The Zwicky Catalog of galaxies (ZC) has been the basis for the Center for Astrophysics (CfA) redshift surveys (Huchra et al. 1996). To date, analyses of the ZC and redshift surveys based on it have relied on heterogeneous sets of galaxy coordinates and redshifts. In order to correct some of the inadequacies of these previous catalogs, Falco et al. (1999) constructed the Updated Zwicky Catalog (UZC), which provides coordinates with $\lesssim 2$ " errors and homogeneously estimated redshifts for the majority of its 19368 galaxies. This catalog is $98 \%$ complete at $m_{\mathrm{Z}} \leq 15.5$ in the CfA 2 region and about $96 \%$ in the remaining northern sky.

It is a well-known fact that the resulting luminosity function is sensitive to the uncertainties of the observational data. In order to control the effect of these uncertainties, we have used throughout this paper the following Montecarlo procedure:

1) We construct a perturbed UZC catalog by introducing, according to their uncertainties, 1- $\sigma$ Gaussian random variations on the central observational data: apparent magnitudes, redshifts, and Hubble types.

2) We correct the perturbed catalog from Galactic extinction, $K$-dimming and virgocentric infall.

3) We select a volume-limited subsample which is complete both in apparent and absolute magnitude.

4) Galaxy groups are identified by using a friends-offriends (FOF) algorithm (Huchra \& Geller 1982) with a percolation parameter of $1.7 \mathrm{Mpc}$ giving, for our subsample, the same density contrast $(\delta \rho / \rho=15)$ as in Nolthenius (1993). We show elsewhere (Cuesta-Bolao \& Serna 2003) that the FOF method gives results which, for the subsamples considered in this paper, are almost identical to those obtained from other algorithms such as the H-method (Materne 1978; Serna \& Gerbal 1996).
5) Using the selected subsample, we compute the luminosity function for different subsets of galaxies selected according to their morphological types and their memberships to groups or clusters. All these LFs are later fitted to the parametric expression proposed by Schechter (1976).

6) Steps (1)-(5) are repeated to obtain $N=1000$ Montecarlo realizations. In this way, the averaged overall and type-dependent luminosity functions are obtained with error bars giving the $1-\sigma$ effect of observational uncertainties. In addition, this procedure provides us with the $1-\sigma$ error ellipses on the Schechter fitting parameters

We will now describe more in detail the way in which some of these steps have been implemented.

\subsection{Uncertainties on the observational data}

The basic data needed to compute type-specific LFs are redshifts (or heliocentric velocities), apparent magnitudes and morphological types.

The Updated Zwicky Catalog provides detailed information on the redshift and its rms error for each individual galaxy. Magnitudes in the UZC are still the original Zwicky magnitudes, which have $\sim 0.3$ mag errors (Falco et al. 1999).

In contrast with its original source catalog (ZCAT), the UZC does not contain any information about the morphological type of galaxies. Since such an information is essential for the purposes of this paper, we have used the Lyon-Meudon Extragalactic Database (LEDA) to obtain both the morphological classification of each galaxy and an estimate of its uncertainty.

These individual errors on redshifts and Hubble types, as well as the generic 0.3 mag error of the Zwicky magnitudes, have been taken into account to construct the 1- $\sigma$ perturbed UZC of each Montecarlo realization.

\subsection{Corrections}

Apparent magnitudes and redshifts have been corrected for Galactic extinction, $K$-dimming and Virgocentric infall.

The correction for Galactic extinction has been carried out by using (Sandage 1973):

$\delta m= \begin{cases}0.132(\csc |b|-1), & |b| \leq 40^{\circ} \\ 0.132\left(\csc 40^{\circ}-1\right)\left(\frac{50^{\circ}-|b|}{10^{\circ}}\right), & 40^{\circ} \leq|b| \leq 50^{\circ} \\ 0, & |b| \geq 50^{\circ}\end{cases}$

while the $K$-dimming has been corrected through the typedependent expression derived by Pence (1976):

$\delta m=10^{-4} c z \times \begin{cases}0.15, & T \leq 0 \\ 0.15-0.025 T & 0 \leq T \leq 3 \\ 0.075-0.01(T-3) & T \geq 3\end{cases}$

where $T$ denotes the morphological type $\left(T_{\mathrm{E} / \mathrm{S} 0} \leq 0, T_{\mathrm{Sa}}=1\right.$, $T_{\mathrm{Sab}}=2, T_{\mathrm{Sb}}=3$, and so on).

For moderate redshifts $\left(c z \lesssim 2600 \mathrm{~km} \mathrm{~s}^{-1}\right)$, these corrections are at most $\delta m=0.2$ so that the effective limiting magnitude is reduced to $m \leq 15.3$. We have checked whether the resulting subsamples were complete at this limiting magnitude by 
computing the average value of $V / V_{m}$ (Schmidt 1968; Huchra \& Sargent 1973), where $V$ is the sample volume between every galaxy and the observer while $V_{m}$ is the volume the galaxy could lie in without dropping below $m_{\mathrm{lim}}$. A homogeneous sample is complete at magnitude $m$ if the average $V / V_{m}$ is close to 0.5 . Here the average $V / V_{m}$ value at $m=15.3$ was in fact close to 0.5 in all Montecarlo realizations.

Heliocentric velocities, $v_{\odot}$, are expressed in the Local Group frame (Sandage et al. 1985):

$v_{\mathrm{LG}}=v_{\odot}+300 \sin l \cos b \mathrm{~km} \mathrm{~s}^{-1}$

and corrected for virgocentric infall using the expression (Schechter 1980):

$v_{\mathrm{obs}}=v_{\mathrm{V}} x-v_{\mathrm{vc}}(\cos \theta-x)\left[1-\left(x^{2}-2 x \cos \theta+1\right)^{-1}\right]$

where $v_{\mathrm{V}}=1097 \mathrm{~km} \mathrm{~s}^{-1}$ is the observed velocity of the Virgo cluster reduced to the centroid of the Local Group; $v_{\mathrm{vc}}=$ $220 \mathrm{~km} \mathrm{~s}^{-1}$ is the infall velocity of our Galaxy toward Virgo; $\theta$ is the angular separation between the Virgo cluster and the galaxy under consideration and $x$ is the distance of a galaxy in units of the Virgo distance. By solving Eq. (4) to obtain the $x$ value, the corrected velocity of the galaxy is given by:

$v=x\left(v_{\mathrm{V}}+v_{\mathrm{vc}}\right)$.

When this solution is not unique, the galaxy belongs to the Virgo cluster.

Galaxy distances are then calculated directly from their corrected velocities (we assume $H_{0}=65 \mathrm{~km} \mathrm{~s}^{-1} \mathrm{Mpc}^{-1}$ throughout this paper).

\subsection{Selection of the galaxy sample}

At each Montecarlo realization, we have extracted a subsample of the perturbed UZC according to the following criteria:

1) In order to minimize the effects of Galactic absorption, we only consider those galaxies with galactic latitudes $|b| \geq 30^{\circ}$. Therefore, our sample covers a solid angle of

$\Omega=\int_{0}^{2 \pi} \int_{\delta_{\min }}^{\pi / 2} f(\alpha, \delta) \cos \delta \mathrm{d} \delta \mathrm{d} \alpha \simeq 3.31 \mathrm{str}$

where $\delta_{\min }=-2.5^{\circ}$ is the smallest declination value of the $\mathrm{UZC}$, while $f$ is a step function equal to 1 (if $|b| \geq 30^{\circ}$ ) or 0 (if $|b|<30^{\circ}$ ).

2) We then extract a volume-limited subsample which is complete both in apparent and absolute magnitude. To that end, we select only those galaxies within a distance $r_{\lim } \mathrm{Mpc}$ and having an absolute magnitude brighter than $M_{\mathrm{lim}}=m_{\mathrm{lim}}$ $25-5 \log r_{\lim }$, with $m_{\lim }=15.3$. In order to chose an appropriate value for $r_{\text {lim }}$, we note that relatively high $r_{\text {lim }}$ values (e.g., $64 \mathrm{Mpc}$ ) yield to subsamples which only cover a small range of absolute magnitudes. In the opposite, relatively small $r_{\lim }$ values (e.g., $32 \mathrm{Mpc}$ ) yield to samples with an excessively small number of galaxies. In this paper, we have considered the fiducial value $r_{\text {lim }}=40 \mathrm{Mpc}$. With this choice for $r_{\text {lim }}$, our subsample typically contains $\sim 900$ galaxies and is distributed as shown in Fig. 1.

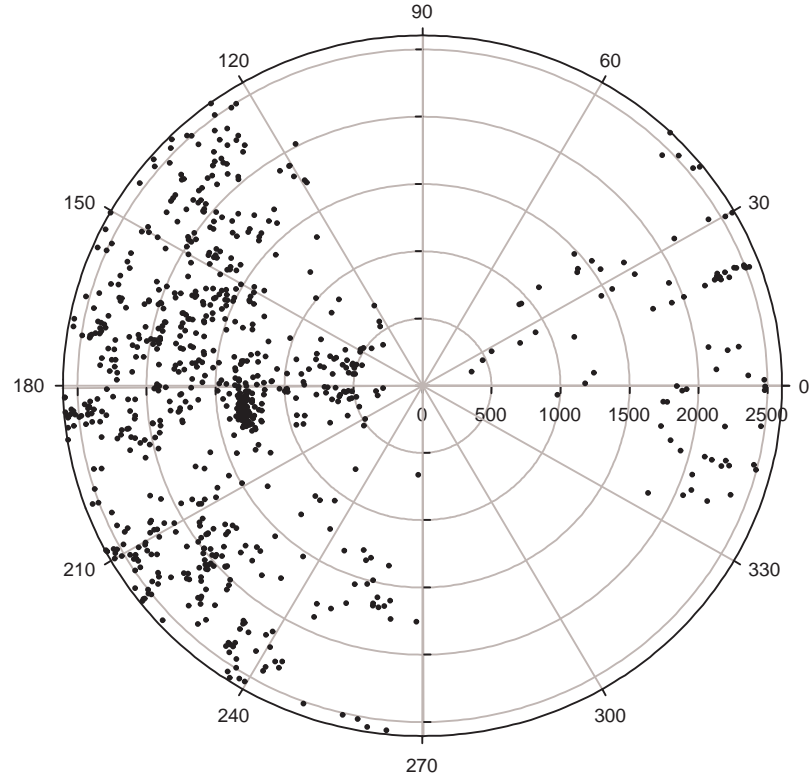

Fig. 1. Distribution of galaxies in the sample.

This selection procedure has the advantage of ensuring that subsamples contain all galaxies intrinsically brighter than $M_{\text {lim }}$ within a fix volume. This allows for an easier comparison with numerical simulations.

\subsection{The luminosity function}

The galaxy luminosity function, $\Phi$, is defined as the number density of galaxies per luminosity unit.

At faint absolute magnitudes, the LF becomes increasingly uncertain due to the incompleteness in absolute magnitudes, to the point where it becomes meaningless. This is inherent to every LF study of field galaxies from apparent magnitudelimited samples. Different methods have been proposed to evaluate the LF by correcting from the incompleteness in absolute magnitudes. The method termed as classical by Binggeli et al. (1987) is based on the assumption that galaxies are uniformly distributed in space. The LF is then computed by counting the numbers of galaxies in bins of $\left(M-\frac{1}{2} \Delta M, M+\frac{1}{2} \Delta M\right)$ and dividing individually these numbers by $V(M)$. Here, $V(M)$ is determined by the maximum distance an object of absolute magnitude $M$ can have and still be in the sample. Since the assumption of homogeneity does not hold for samples with a small limiting redshift, other non-parametric methods have been proposed to compute the LF without any assumption on the spatial distribution of galaxies. For example, the so-called C-method (Lynden-Bell 1971; Choloniewski 1987) or methods based on maximum likelihood techniques (Nicol \& Segal 1983; Efstathiou et al. 1988).

Since our galaxy subsamples are specifically constructed to be complete in both apparent and absolute magnitudes, all the above methods give identical results. In Appendix A we show in fact that, for this kind of samples, the classical and $C$ methods are absolutely equivalent. Due to its simplicity, we have then used the classical method to estimate the observed LFs. 
In order to quantify the shape of the measured LFs, they have been later fitted to a parametric analytical expression. The most popular and often used expression to parameterize the observed LF was introduced by Schechter (1976):

$\Phi=\phi_{*} 10^{0.4\left(M_{*}-M\right)(1+\alpha)} \exp \left(-10^{0.4\left(M_{*}-M\right)}\right)$

where $\phi_{*}$ is the normalization, whereas $M_{*}$ and $\alpha$ are the shape parameters. The $\alpha$ parameter represents the logarithmic slope of $\Phi$ at faint magnitudes. An $\alpha$ value more negative than -1 implies a luminosity function with an increasing faint-end, whereas $\alpha>-1$ implies a decreasing logarithmic slope. The limiting value $\alpha=-1$ corresponds to a flat faint-end of $\Phi$. The $M_{*}$ parameter is sometimes termed as the "knee" magnitude. It represents a characteristic magnitude which separates the exponential and power law behaviors of $\Phi$. For absolute magnitudes much brighter than $M_{*}$, the LF is dominated by the exponential growth.

\section{Luminosity functions of field galaxies}

We will first evaluate the luminosity function of the nearby field galaxies. As it is usual in this kind of studies, by "field" we mean all the galaxies in the subsample, either isolated or in groups. The Virgo cluster is nevertheless excluded from the field subsamples to allow for the possibility that the local field can represent an arbitrary field in the universe.

\subsection{The overall luminosity function}

Figure 2 and Table 1 show our results for the LF of field galaxies of all morphological types. The corresponding $1 \sigma$-error ellipse is shown in Fig. 3. We see that the faint-end of the overall LF is nearly flat $(\alpha=-0.95 \pm 0.07)$. The Schechter function provides a good fit to the observed LF, and we do not observe any apparent excess of bright galaxies.

Although we discuss below the specific results for E/S0 and S/I galaxies, we already note in Fig. 2 that S/I galaxies are more abundant than $\mathrm{E} / \mathrm{S} 0$ galaxies in a wide range of absolute magnitudes. Within the $M$ range considered in this paper, E/S0 galaxies are dominant only for absolute magnitudes brighter than $\sim-21.5$.

\subsection{The luminosity function of $\mathrm{E} / \mathrm{SO}$ galaxies}

We show in Table 1 and Fig. 4 our results for the LF of $E$ and $\mathrm{S} 0+\mathrm{S} 0$ a galaxies. The Schechter parameters for E/S0 galaxies are not very different from those found for the overall sample. In particular, we obtain $\alpha=-0.88 \pm 0.13$ implying a slightly decreasing faint-end of the E/S0 LF. Within $1 \sigma$ uncertainties, such an $\alpha$ value is also compatible with a flat faint-end, while excludes a very steeply decreasing or increasing behavior of the luminosity function of faint E/S0 galaxies. On the other hand, the $M_{*}$ value for $\mathrm{E} / \mathrm{S} 0$ galaxies $(-20.55 \pm 0.20)$ is only slightly brighter (by about $0.2 \mathrm{mag}$ ) than that obtained for the total sample.

When E and S0 galaxies are considered separately, we find clear evidences that their LFs are considerably different. As a

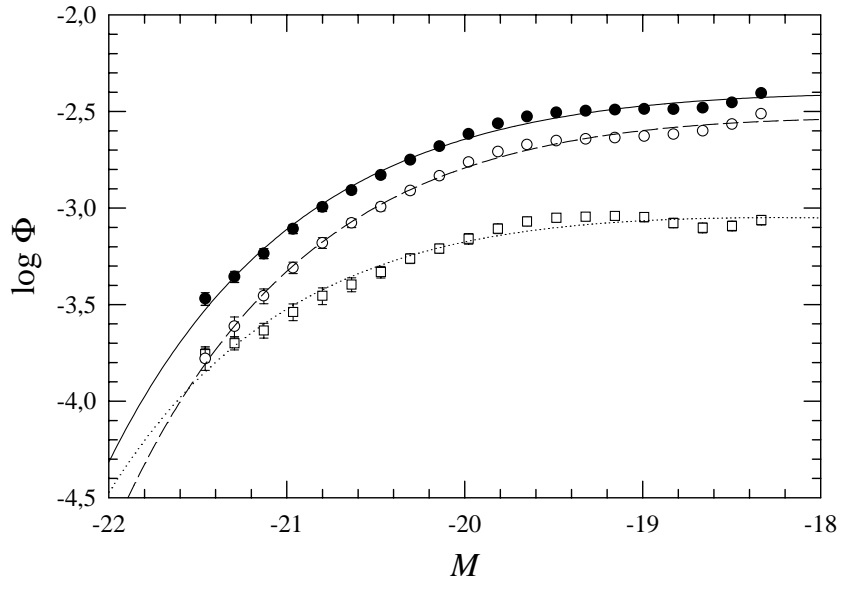

Fig. 2. Luminosity function for all galaxies in the sample. The observational data are represented by filled circles (all types), open squares $(\mathrm{E}+\mathrm{S} 0)$ and open circles $(\mathrm{S}+\mathrm{I})$ with $1-\sigma$ error bars obtained from Montecarlo simulations. The best Schechter fits are represented by solid, dotted, and dashed lines, respectively.

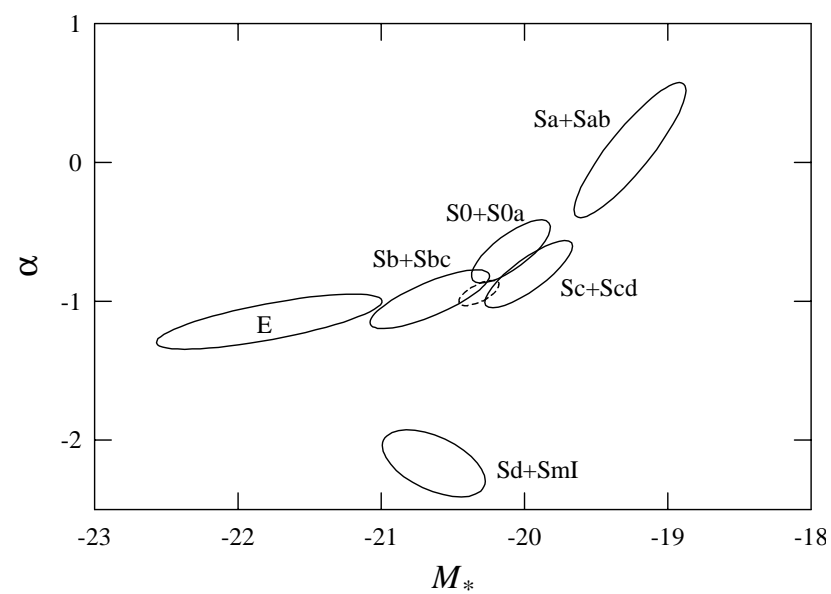

Fig. 3. Ellipses of 1- $\sigma$ errors on the Schechter parameters obtained for different morphological types. The dashed ellipse represents the error ellipse for the overall sample.

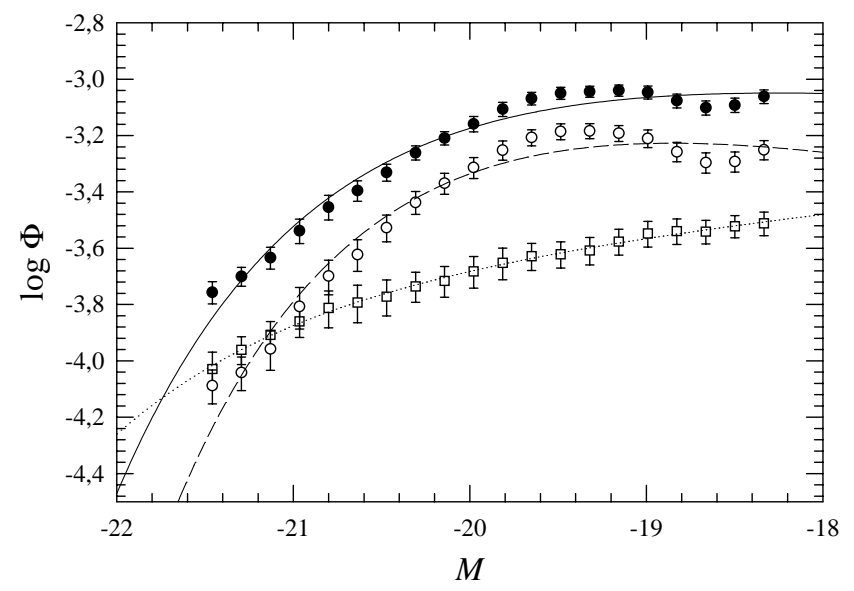

Fig. 4. Luminosity function for elliptical and S0 galaxies. The observational data are represented by filled circles $(\mathrm{E}+\mathrm{S} 0)$, open circles (S0) and open squares (E) with 1- $\sigma$ errors obtained from Montecarlo simulations. The best Schechter fits are represented by the solid, dashed and dotted lines, respectively. 
Table 1. Best Schechter fits for field galaxies.

\begin{tabular}{lrccc}
\hline \multicolumn{1}{c}{ Type } & $\langle N\rangle$ & $\phi_{*} / 10^{-3}$ & $M_{*}$ & $\alpha$ \\
\hline All & 707 & $4.8 \pm 0.5$ & $-20.32 \pm 0.10$ & $-0.95 \pm 0.07$ \\
& \multicolumn{4}{c}{ Coarse-grained type bins } \\
E/S0 & 187 & $1.3 \pm 0.3$ & $-20.55 \pm 0.20$ & $-0.88 \pm 0.13$ \\
S/I & 520 & $3.9 \pm 0.6$ & $-20.16 \pm 0.15$ & $-0.92 \pm 0.10$ \\
& \multicolumn{4}{c}{ Standard type bins } \\
E & 62 & $0.2 \pm 0.1$ & $-21.78 \pm 0.90$ & $-1.16 \pm 0.20$ \\
S0+S0a & 125 & $1.2 \pm 0.3$ & $-20.14 \pm 0.25$ & $-0.69 \pm 0.20$ \\
Sa+Sab & 88 & $1.1 \pm 0.2$ & $-19.69 \pm 0.36$ & $-0.43 \pm 0.45$ \\
Sb+Sbc & 150 & $0.9 \pm 0.3$ & $-20.67 \pm 0.39$ & $-0.99 \pm 0.19$ \\
Sc+Scd & 212 & $2.0 \pm 0.5$ & $-19.91 \pm 0.29$ & $-0.75 \pm 0.22$ \\
Sd+I & 70 & $0.1 \pm 0.0$ & $-20.67 \pm 0.32$ & $-2.15 \pm 0.20$ \\
& \multicolumn{4}{c}{ Fine type bins } \\
E & 62 & $0.2 \pm 0.1$ & $-21.78 \pm 0.90$ & $-1.16 \pm 0.20$ \\
S0 & 89 & $1.1 \pm 0.2$ & $-19.87 \pm 0.32$ & $-0.43 \pm 0.31$ \\
S0a & 36 & $0.1 \pm 0.1$ & $-21.52 \pm 1.30$ & $-1.37 \pm 0.54$ \\
Sa & 47 & $0.7 \pm 0.2$ & $-19.52 \pm 0.45$ & $-0.11 \pm 0.56$ \\
Sab & 41 & $0.4 \pm 0.2$ & $-20.10 \pm 0.55$ & $-0.82 \pm 0.53$ \\
Sb & 79 & $0.3 \pm 0.2$ & $-21.03 \pm 0.74$ & $-1.29 \pm 0.31$ \\
Sbc & 71 & $0.6 \pm 0.2$ & $-20.51 \pm 0.61$ & $-0.76 \pm 0.31$ \\
Sc & 171 & $1.9 \pm 0.4$ & $-19.85 \pm 0.26$ & $-0.60 \pm 0.21$ \\
Scd & 41 & $0.1 \pm 0.1$ & $-21.29 \pm 1.00$ & $-1.94 \pm 0.50$ \\
Sd & 25 & $0.1 \pm 0.1$ & $-22.91 \pm 0.43$ & $-2.40 \pm 0.60$ \\
I & 45 & $0.1 \pm 0.1$ & $-20.99 \pm 0.40$ & $-2.12 \pm 0.25$ \\
\hline & & & &
\end{tabular}

matter of fact, we can see from Fig. 3 (see also Table 1) that the faint-end of elliptical galaxies increases $(\alpha=-1.16 \pm 0.20)$, while that of lenticular galaxies decreases $(\alpha=-0.69 \pm 0.20)$. In addition, the $M_{*}$ value for E galaxies is considerably brighter (by about $1.6 \mathrm{mag}$ ) than that found for $\mathrm{S} 0$ galaxies. As a consequence, the bright end of the E/S0 LF is clearly dominated by E galaxies (see Fig. 4), while S0 galaxies become more abundant than ellipticals at $M$ values fainter than $\sim-21.2$.

\subsection{The luminosity function of $S / I$ galaxies}

Our analysis for the UZC implies (see Fig. 5 and Table 1) a luminosity function of S/I galaxies with a $M_{*}$ parameter slightly fainter (by about $0.15 \mathrm{mag}$ ) than $M_{*}^{\text {total }}$ and a moderately decreasing faint-end ( $\alpha=-0.92 \pm 0.10)$ which is compatible with a flat logarithmic slope within $1 \sigma$ uncertainties.

We have also analyzed the LF of spiral and irregular galaxies by considering different morphological bins. Firstly, we have considered four morphological groups: $\mathrm{Sa}+\mathrm{Sab}, \mathrm{Sb}+\mathrm{Sbc}$, $\mathrm{Sc}+\mathrm{Scd}$ and $\mathrm{Sd}+\mathrm{I}$ galaxies. Their corresponding LFs are displayed in Fig. 5, whereas their Schechter parameters and $1 \sigma$ error ellipses are shown in Table 1 and Fig. 3. We find that the $\alpha$ parameter exhibits a significant decrease from early to late $\mathrm{S} / \mathrm{I}$ galaxies. The $\mathrm{LF}$ of early spirals $(\mathrm{Sa}+\mathrm{Sab})$ has in fact a steeply decreasing faint-end $(\alpha=-0.43 \pm 0.45)$ while that of $\mathrm{Sd}+\mathrm{I}$ galaxies has a steeply increasing logarithmic slope $(\alpha=-2.15 \pm 0.20)$. The intermediate spiral types $(\mathrm{Sb}+\mathrm{Sbc}$ and

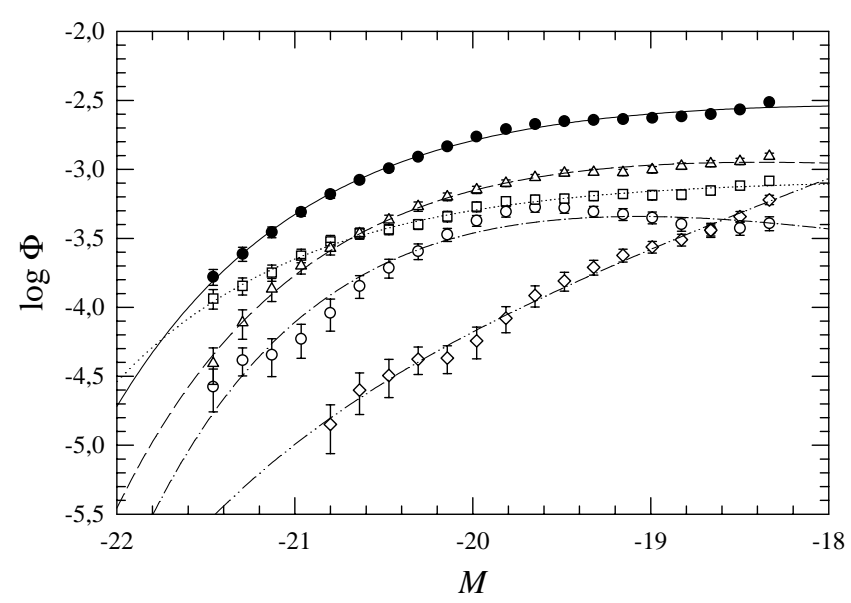

Fig. 5. Luminosity function for $\mathrm{S} / \mathrm{I}$ galaxies. The observational data are represented by filled circles (all spirals), open circles $(\mathrm{Sa}+\mathrm{Sab})$, squares $(\mathrm{Sb}+\mathrm{Sbc})$, triangles $(\mathrm{Sc}+\mathrm{Scd})$ and diamonds $(\mathrm{Sd}+\mathrm{I})$, with $1-\sigma$ errors obtained from Montecarlo simulations. The lines represent the best Schechter fits.

$\mathrm{Sc}+\mathrm{Scd}$ ) have $\alpha$ values which are not very different from that obtained for the overall sample. Taking into account the $1 \sigma$ uncertainties, the LFs of such intermediate spiral types are compatible with a flat faint-end. Although less evident, a trend is also observed for the $M_{*}$ parameter: its value for very early spiral types $(\mathrm{Sa}+\mathrm{Sab})$ appears to be significantly fainter (by about $1 \mathrm{mag}$ ) than for $\mathrm{Sd}+\mathrm{I}$ galaxies.

These trends for the $\alpha$ and $M_{*}$ values are also observed when fine type bins are considered (see Table 1). However, the Schechter parameters have now large uncertainties due to poor number statistics. The LF of Sa galaxies has a steeply decreasing faint-end, Sab to Sc spirals have a moderately decreasing or increasing logarithmic slope, and Scd to I objects have LFs with a steeply increasing faint-end.

\section{The luminosity function of group galaxies}

We will now investigate the environmental dependence of the LF of galaxies within groups and clusters. As we have already mentioned in Sect. 2, galaxy groups and clusters have been identified in each Montecarlo realization by means of a friendsof-friends algorithm.

The resulting galaxy groups have numbers which range from $N=3$ (the minimum required to be considered as a galaxy group) to $N \bumpeq 165$ (the Virgo cluster). We have performed an exhaustive analysis of the different possibilities for distributing these groups into bins, according to the number of members. We have found that very fine bins are not necessary because the environmental dependence of the LFTs is not very strong. In addition, very fine bins are affected by large uncertainties due to small number statistics. Consequently, we have finally chosen the following group binning: a) Small groups, which include from triplets to groups with $N \leq 7$ galaxies brighter than our limiting absolute magnitude $M=-17.7$; b) Large groups, with $N \geq 8$; c) Poor clusters, which refer to the Ursa Major cluster; and d) Moderately rich clusters, which refer to the Virgo cluster. 
Table 2. Best Schechter fits for isolated and group galaxies.

\begin{tabular}{lrrrcc}
\hline \hline Type & $\langle N\rangle$ & $\%$ & $\phi_{*}$ & $M_{*}$ \\
\hline \multicolumn{5}{c}{ Isolated } \\
All & 349 & $38.9^{*}$ & 1.00 & $-20.57 \pm 0.29$ & $-1.21 \pm 0.15$ \\
E/S0 & 77 & 22.1 & 0.35 & $-20.40 \pm 0.45$ & $-0.91 \pm 0.31$ \\
S/I & 272 & 77.9 & 0.69 & $-20.59 \pm 0.38$ & $-1.29 \pm 0.18$ \\
\multicolumn{5}{c}{ Small groups $(N \leq 7)$} \\
All & 191 & $21.3^{*}$ & 1.00 & $-20.34 \pm 0.27$ & $-0.78 \pm 0.19$ \\
E/S0 & 61 & 31.9 & 0.28 & $-20.86 \pm 0.67$ & $-0.82 \pm 0.32$ \\
S/I & 130 & 68.1 & 0.78 & $-20.10 \pm 0.36$ & $-0.73 \pm 0.29$ \\
\multicolumn{5}{c}{ Large groups $(N \geq 8)$} \\
All & 146 & $16.2^{*}$ & 1.00 & $-19.98 \pm 0.36$ & $-0.68 \pm 0.28$ \\
E/S0 & 44 & 30.1 & 0.25 & $-20.31 \pm 0.60$ & $-0.85 \pm 0.47$ \\
S/I & 102 & 69.9 & \multicolumn{5}{c}{$-19.87 \pm 0.40$} & $-0.59 \pm 0.31$ \\
& \multicolumn{5}{c}{ Poor clusters (Ursa Major) } \\
All & 47 & $5.2^{*}$ & 1.00 & $-20.53 \pm 1.10$ & $-0.81 \pm 0.45$ \\
E/S0 & 12 & 25.5 & 0.03 & $-24.42 \pm 1.62$ & $-1.34 \pm 0.24$ \\
S/I & 35 & 74.5 & 0.79 & $-20.55 \pm 0.92$ & $-0.76 \pm 0.38$ \\
All & 165 & $18.4^{*}$ & 1.00 & $-21.01 \pm 0.32$ & $-1.38 \pm 0.11$ \\
E/S0 & 79 & 47.9 & 0.22 & $-21.38 \pm 0.32$ & $-1.67 \pm 0.16$ \\
S/I & 86 & 52.1 & 0.86 & $-20.76 \pm 0.17$ & $-1.10 \pm 0.06$ \\
\hline Referately rich clusters (Virgo)
\end{tabular}

${ }^{*}$ Refereed to the overall sample.

Our analysis does not include very rich clusters (e.g., Coma) because the local $(c z \leq 2600)$ northern field only contains the two mentioned clusters, Ursa Major and Virgo. Nevertheless, as we will see bellow, the richness range considered in this paper is large enough to obtain significant conclusions.

\subsection{Cluster galaxies}

Table 2 and the upper panel of Fig. 6 show our results for the Virgo luminosity function. We see that the Virgo LF has substantial differences with respect to that measured for field galaxies. The most remarkable difference is the logarithmic slope of the faint-end, which exhibits an increasing behavior ( $\alpha=-1.38$ ) instead of the flat faint-end found for the overall LF of field galaxies. In addition, the $M_{*}$ value for the Virgo cluster is significantly brighter (by about $0.7 \mathrm{mag}$ ) than that observed for the local field. Another remarkable difference is that the Virgo E/S0 galaxies are not dominant at the very bright magnitudes and become more abundant than spirals for absolute magnitudes fainter than a relatively bright value $M \simeq-19$. As a consequence, the observed faint-end of the overall LF for the Virgo cluster is essentially due to the steeply increasing behavior of elliptical galaxies.

The lower panel of Fig. 6 shows our results for the Ursa Major LF, which we believe representative of very poor clusters. We find that Ursa Major has a galaxy distribution very different from that found for the Virgo cluster. Instead of the increasing logarithmic slope observed for the Virgo cluster,
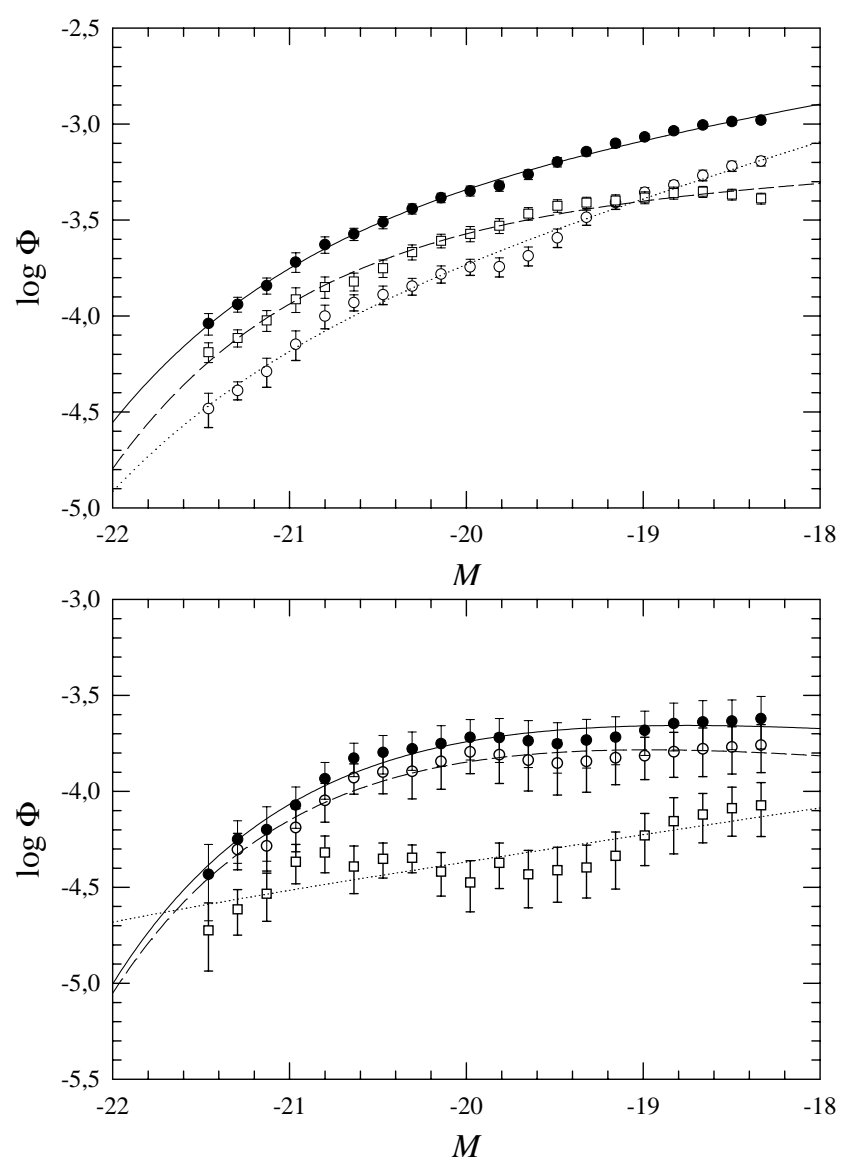

Fig. 6. Luminosity function for the Virgo (upper panel) and Ursa Major (lower panel) clusters. The observational data are represented by filled circles (all galaxies), open circles ( $\mathrm{S}+\mathrm{I}$ galaxies) and open squares (E+S0 galaxies) with 1- $\sigma$ errors obtained from Montecarlo simulations. The lines represent the best Schechter fits.

the Ursa Major LF has a slightly decreasing faint-end ( $\alpha=$ $-0.81 \pm 0.45)$.

\subsection{Group galaxies}

Figure 8 and Table 2 show our results for the LFs of group galaxies. We find that both small and large groups have LFs with a moderately decreasing logarithmic slope. Elliptical galaxies are more abundant than spirals at absolute magnitudes brighter than $\sim-21.5$ (in large groups) or $\sim-21.0$ (in small groups), whereas the faint-end is dominated by spiral galaxies (up to our limiting magnitude -17.7 ).

The inspection of the error ellipses (Fig. 7) shows that the overall LF of small groups is indistinguishable from that observed for large groups. In the same way, S/I galaxies in small and large groups have indistinguishable LFs. The main difference between these two kinds of groups arises from E/S0 galaxies, where the $M_{*}$ value of small groups is significantly brighter than that found for large groups.

It must be also noted in the upper panel of Fig. 7 that the luminosity functions for small and large galaxy groups are close to that observed for very poor clusters. Moderately rich clusters and isolated galaxies have instead considerably different LFs. 

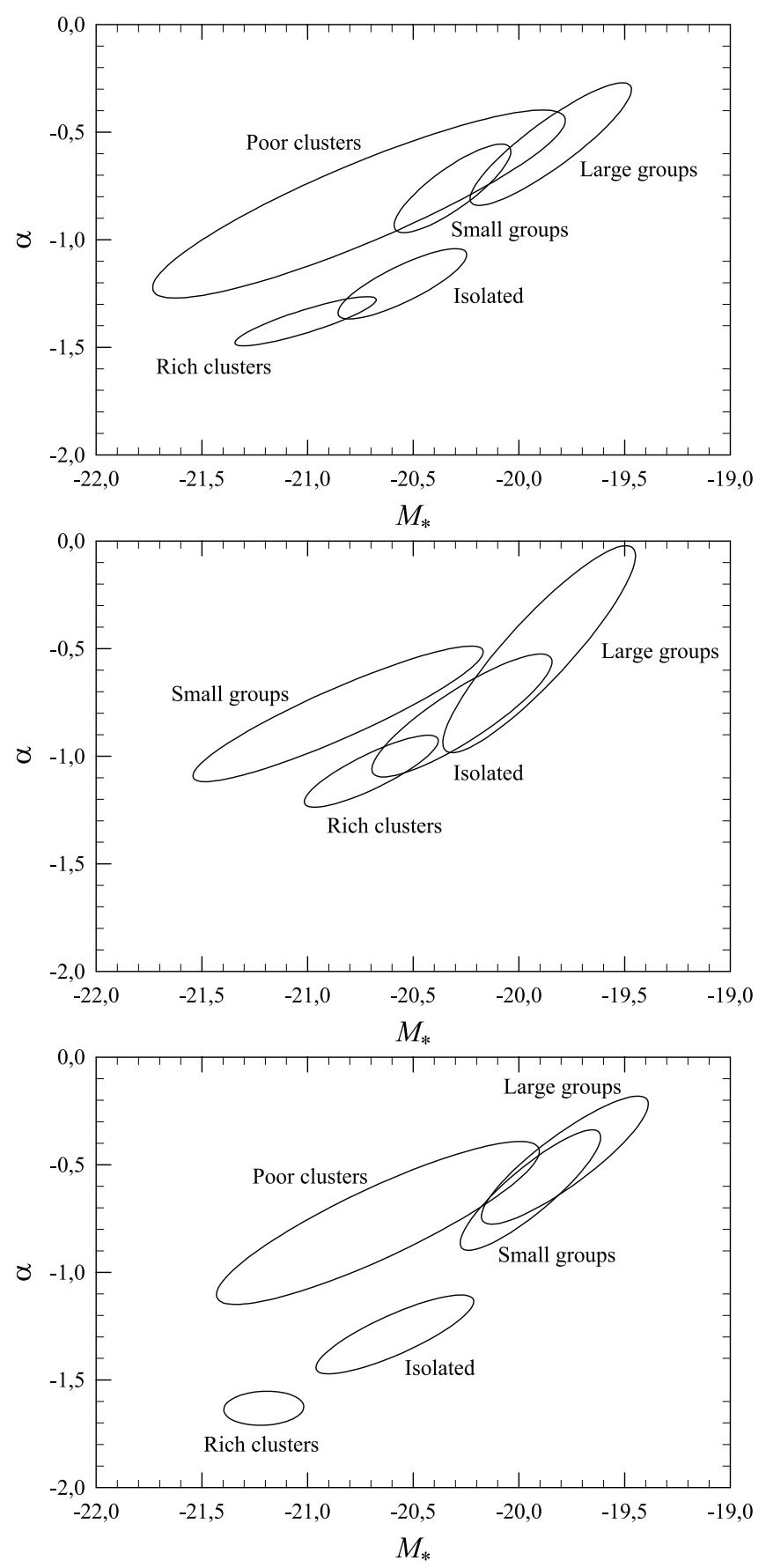

Fig. 7. Ellipse of $1-\sigma$ errors on the Schechter parameters obtained in different environments for all morphological types (upper panel), $\mathrm{E}+\mathrm{S} 0$ (central panel) and S+I (lower panel) galaxies.

\subsection{Isolated galaxies}

We finally show (Fig. 9) the LF of isolated galaxies, defined as those which do not belong to any friend-of-friend group. We find remarkable differences with respect to the LFs observed in other environments. In particular, the LF of isolated galaxies has a moderately increasing faint-end $(\alpha=-1.21 \pm 0.15)$ and it is dominated at any $M$ value by $\mathrm{S} / \mathrm{I}$ galaxies.

An interesting fact observed in Fig. 7 is that both the overall and E/S0 luminosity functions of isolated galaxies are indistinguishable from those observed in moderately rich clusters. In
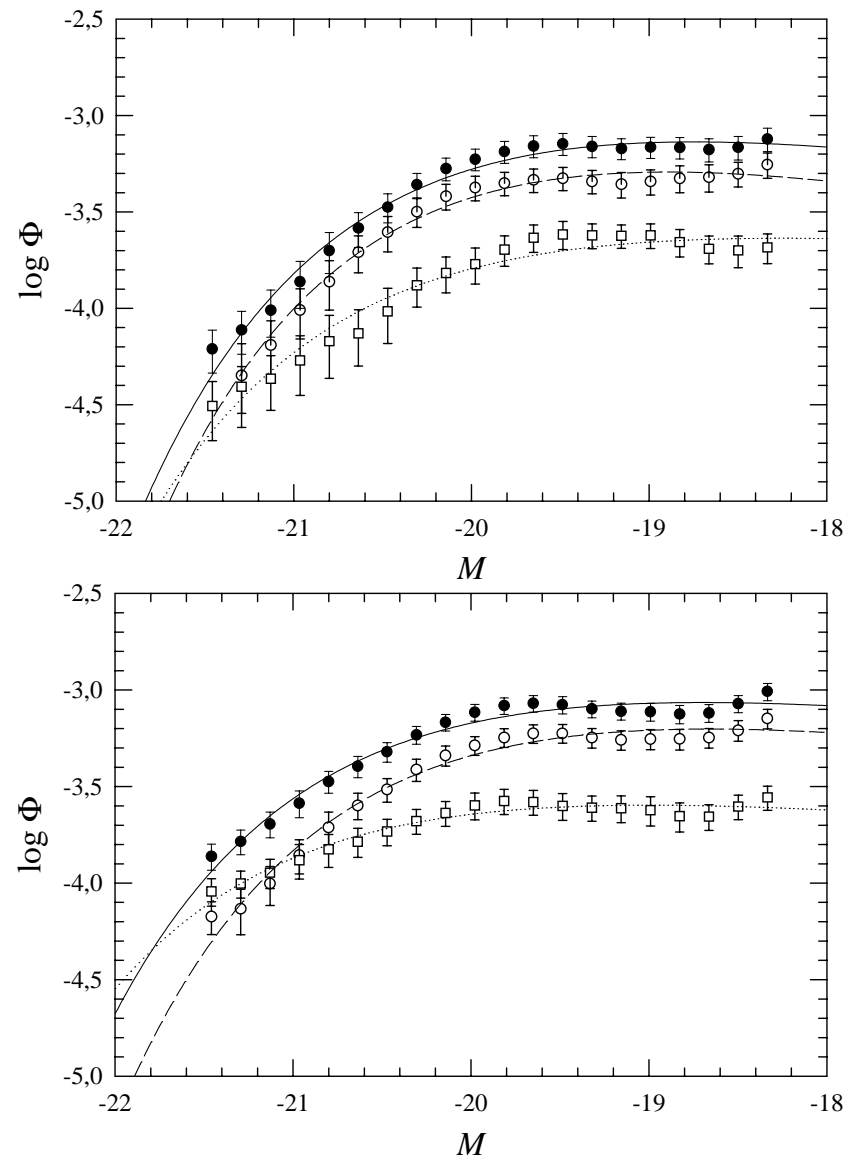

Fig. 8. Luminosity function for large groups (upper panel) and small groups (lower panel). The observational data are represented by filled circles (all galaxies), open circles ( $\mathrm{S}+\mathrm{I}$ galaxies) and open squares (E+S0 galaxies) with 1- $\sigma$ errors obtained from Montecarlo simulations. The lines represent the best Schechter fits.

the opposite, isolated S/I galaxies have a LF significantly different from that measured for $\mathrm{S} / \mathrm{I}$ galaxies in rich clusters.

\section{The universality of LFTs}

We will finally address the question of the possible universality of the LFTs. Although the results reported in Sect. 4 already suggest an environmental dependence of the LFTs, more robust tests are needed.

In order to analyze the hypothesis of specific LFTs with universal shapes, independently of the environment, we will perform the following test. If we accept such a hypothesis, then we could use the LFTs obtained in Sect. 3 to predict the overall LF of any cluster or environment with a known morphological composition.

More specifically, if we accept the hypothesis that the shape of the luminosity function is only a function of the Hubble type, $\Phi(T)$, the specific LFT of each morphological type $i$ could be expressed as:

$\Phi_{i}(M)=\phi_{*, i} \phi\left(T_{i}, M\right)$ 


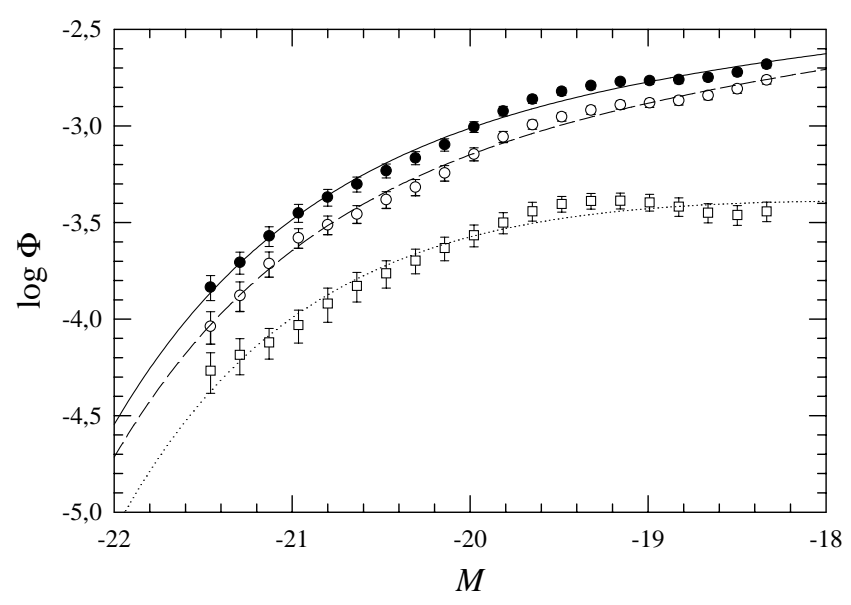

Fig. 9. Luminosity function for isolated galaxies. The observational data are represented by filled circles (all galaxies), open circles ( $\mathrm{S}+\mathrm{I}$ galaxies) and open squares (E+S0 galaxies) with $1-\sigma$ errors obtained from Montecarlo simulations. The lines represent the best Schechter fits.

where $\phi\left(T_{i}, M\right)$ would denote the universal shape of the $i$ type LF:

$\phi\left(T_{i}, M\right)=10^{0.4\left(M_{*, i}-M\right)\left(1+\alpha_{i}\right)} \exp \left(-10^{0.4\left(M_{*, i}-M\right)}\right)$.

The $i$-type LFTs of different clusters would only differ in their normalization constants $\phi_{*, i}$. If the morphological composition of a cluster is known, down to $M_{\text {lim }}$ (in this paper $M_{\mathrm{lim}}=-17.7 \mathrm{mag}$ ), the $\phi_{*, i}$ values are fixed by the condition that

$N_{i}=\phi_{*, i} V_{\mathrm{t}} \int_{-\infty}^{M_{\lim }} \phi\left(T_{i}, M\right) \mathrm{d} M$

must give the observed number $N_{i}$ of $i$-type galaxies brighter than $M_{\text {lim }}$ within the sampled volume $V_{\mathrm{t}}{ }^{1}$. Here, the integral

$I_{i}=\int_{-\infty}^{M_{\lim }} \phi\left(T_{i}, M\right) \mathrm{d} M$

would be a "universal" constant for each morphological type.

The cluster LF could be then expressed as:

$\Phi(M)=\sum_{i} \phi_{* i} \Phi\left(T_{i}, M\right)=\sum_{i} \frac{N_{i}}{I_{i} V_{\mathrm{t}}} \Phi\left(T_{i}, M\right)$

where all terms are known.

This predicted LF can be directly compared with that actually observed for a cluster (using the same arbitrary $V_{\mathrm{t}}$ value). If the assumption of universal LFTs is correct, the predicted and observed LFs must coincide within their respective uncertainties.

\footnotetext{
${ }^{1}$ In UZC samples as those described in Sect. 2.3, $V_{\mathrm{t}}$ is given by $V_{\mathrm{t}}=(\Omega / 3) r_{\text {lim }}^{3}$. However, in the study of an individual galaxy group, the choice of $V_{\mathrm{t}}$ is somewhat arbitrary. We have used in this latter case an sphere of radius $r_{1}$, where $r_{1}$ is the radial distance of the most distant member.
}

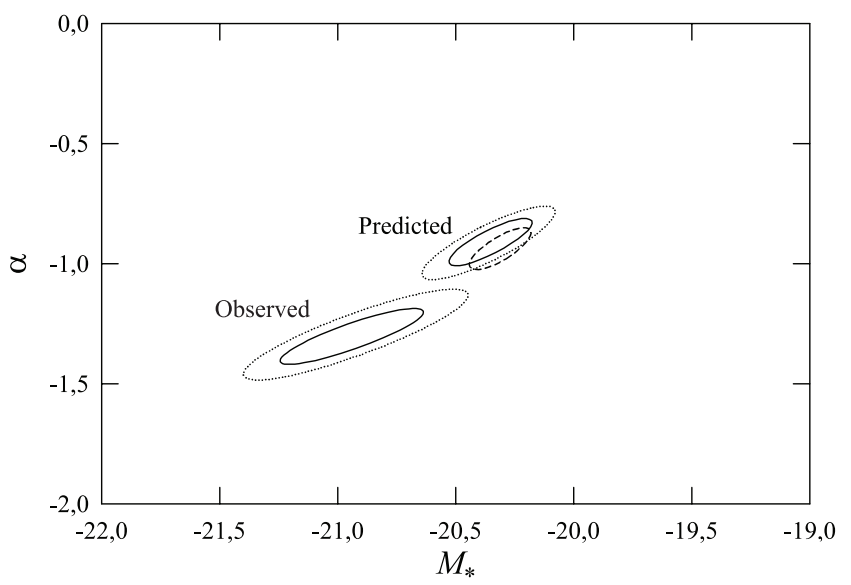

Fig. 10. Observed Schechter parameters for the Virgo cluster as compared to that predicted by the hypothesis of universal LFTs. Solid and dotted lines represent $1 \sigma$ and $3 \sigma$ uncertainties, respectively. The dashed ellipse represents the $1 \sigma$ errors on the Schechter parameters for the overall UZC sample.

\subsection{Importance of the environment}

The above test has been applied for the Virgo cluster by using the LFT shapes summarized in Table 1 (with the finest type binning) and the $N_{i}$ values directly measured for Virgo at each Montecarlo realization. Figure 10 shows the error ellipses for both the observed and predicted shapes of the Virgo luminosity function. We see that the predicted LF is clearly different from that actually observed for this cluster. Consequently, the starting hypothesis of universal LFTs cannot be accepted. As a matter of fact, the predicted LF for Virgo remains close to that found for the UZC local field, which was used to obtain the specific shapes of the LFTs. This result clearly evidences that environmental effects are important and cannot be neglected.

Similar results were obtain by using a less fine type binning. As a matter of fact, the predicted error ellipse for Virgo was in this case almost identical to that shown in Fig. 10.

\subsection{The universal $\Phi(\rho, T)$ function}

Once shown that the luminosity function of galaxies depends both on morphology and environment, we will now analyze whether we can define a universal $\Phi(\rho, T)$ with coarse grained bins both for $\rho$ and $T$. For example, by just distinguishing two morphological bins $(\mathrm{E}+\mathrm{S} 0$ and $\mathrm{S}+\mathrm{I})$ and the five environmental bins used in Sect. 4. This leads to ten galaxy "types": five environmental "types" of E+S0 galaxies, together with their counterparts for $\mathrm{S}+\mathrm{I}$ galaxies (see Fig. 7).

As before, if we accept the hypothesis that these galaxy "types" have luminosity functions $\Phi(\rho, T)$ with universal shapes, we must be able to use these functions to predict the overall LF of any group or cluster with a known morphological composition.

To perform such a test, we have considered a galaxy group classified in Table 2 as a large group $(N \geq 8)$. In particular, one of the largest $(N \sim 25)$ of these groups, identified as that having NGC 4261 as one of its central galaxies. The predicted error ellipse for this group has been computed as in the previous 


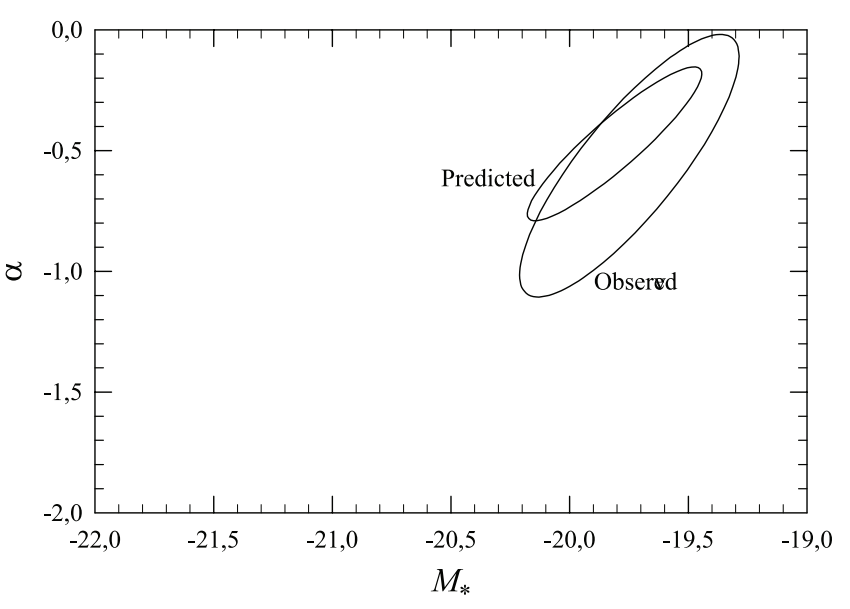

Fig. 11. Observed Schechter parameters for the NGC 4261 group as compared to that predicted by the hypothesis of universal $\phi(\rho, T)$ shape functions.

subsection, but with the ten galaxy "types" defined according to the environment and morphology. In order to avoid any bias favoring the starting hypothesis, the shape function $\phi\left(T_{i}, M\right)$ of "large groups" has been computed by excluding the NGC 4261 group. Figure 11 shows the error ellipses for both the observed and predicted shapes of the group luminosity function. We see that, now, the predicted LF is indistinguishable from that actually observed for this group. Consequently, this result is compatible with the starting hypothesis of universal $\phi(\rho, T)$ shape functions.

\section{Discussion and comparison with previous work}

\subsection{The overall luminosity function}

In Table 3 we show the Schechter parameters (as well as their $1 \sigma$ errors) previously obtained by other authors from various galaxy samples. All these values have been reduced to $H_{0}=$ $65 \mathrm{~km} \mathrm{~s}^{-1} \mathrm{Mpc}^{-1}$ and transformed to the Zwicky photometric system through simple offsets: $B-m_{\mathrm{Z}}=-0.3, B-b_{J}=-0.3$ (Efstathiou et al. 1988), $b_{J}-R=+1.3, r-R=0.35$ (Geller et al. 1997), $b_{J}-K=4.25$ (Mobasher et al. 1993) and $b_{j}-B(0)=0$ (da Costa et al. 1994). It must be however emphasized that such a homogenization procedure introduces uncertainties of about $0.5 \mathrm{mag}$ in $M_{*}$ (Marinoni et al. 1999). In addition, a meaningful discrepancy between the results obtained from different authors can also arise from many other sources: observational selection criteria, methods used in computing the luminosity function, corrections of observed magnitudes and redshifts, cosmological model, completeness, etc.

Inspection of Table 3 shows that, with the exception of Lin et al. (1996), all previous studies of the local field obtain a nearly flat faint-end for the overall LF of galaxies: $0.9 \lesssim \alpha \lesssim 1.15$, in agreement with our results. Deep field galaxy surveys find instead a steeply increasing faint-end for the optical LF of galaxies. This latter result is commonly explained (Mobasher et al. 1993; Ellis et al. 1996) from the fact that deep number-counts in optical wavebands are sensitive to both cosmological parameters and the rate of galaxy luminosity evolution. As a matter of fact, the analysis carried out by
Ellis et al. (1996) of the galaxy LF as a function of redshift (see also Table 3) evidenced a steepening of the faint-end slope with increasing redshift. Some authors (Mobasher et al. 1993; Gardner et al. 1997) have then suggested that a more straightforward measurement of the galaxy LF of deep surveys is provided by infrared wavelengths, where the evolutionary corrections in luminosity are small and possibly better understood than in other wavelengths. Such $K$-band measurements of the LF of galaxies agree in finding a flat faint-end slope (Table 3) similar to that observed for the local field.

We also see from Table 3 that, with the exception of Marzke et al. (1994a,b), most previous studies of the local LF of galaxies find $-20.5 \lesssim M_{*} \lesssim-20.1$ and $0.003 \lesssim \phi \lesssim 0.006 \mathrm{Mpc}^{-3}$. Such values are in reasonable agreement with those obtained in this paper $\left(M_{*}=-20.32 \pm 0.10, \phi=0.0048 \pm 0.0005 \mathrm{Mpc}^{-3}\right)$ as well as with those obtained in deep K-surveys. Deep optical surveys find instead a slightly brighter $M_{*}$ value $(-20.50 \pm 0.25)$ and a slightly higher normalization $\left(\phi=0.006 \pm 0.002 \mathrm{Mpc}^{-3}\right)$.

\subsection{Type-specific luminosity functions}

\subsubsection{The luminosity function of $\mathrm{E} / \mathrm{SO}$ galaxies}

Table 4 summarizes the results found in other works analyzing the LF of both E/S0 and S/I galaxies. We see that, with the exception of Loveday et al. (1992), all other authors obtain a flat $(\alpha \simeq-1)$ or a slightly decreasing $(\alpha \simeq-0.9)$ faint-end of the E/S0 LF in different galaxy surveys. Zucca et al. (1994) and Marzke et al. (1998) suggested that strong deviations from a flat faint-end of the field E/SO LF could be due to the incorrect identification of early-type galaxies at large distances and/or faint magnitudes. This suggestion is supported by our results (see Table 1 and Fig. 4) where the $\alpha$ parameter of E/S0 galaxies implies a slightly decreasing logarithmic slope. Taking into account the error ellipse of E/S0 galaxies (Fig. 3), our results are also compatible with a flat faint-end and exclude a steeply decreasing ( $\alpha \gtrsim-0.6$ ) behavior of the luminosity function of faint E/SO galaxies.

We also see from Table 4 that most previous works obtain, for E/S0 galaxies, a $M_{*}$ value slightly brighter than that obtained for the overall sample, in agreement with our results. In addition, when elliptical and lenticular galaxies are analyzed separately, significant differences are found with respect to the overall sample. The $M_{*}$ value of S0 galaxies usually appears to be considerably fainter than that observed for E galaxies (see Table 4). Although such differences are apparently more pronounced in our results, one must note the large uncertainties (see Fig. 3) specially in the $M_{*}$ value of E galaxies.

\subsubsection{The luminosity function of $S / I$ galaxies}

As we have already mentioned in Sect. 3.3, our analysis for the UZC implies a luminosity function of S/I galaxies with a $M_{*}$ parameter slightly fainter (by about $0.15 \mathrm{mag}$ ) than $M_{*}^{\text {total }}$ and a nearly flat logarithmic slope. Such a result is in reasonable agreement with those previously found in other works (see Table 4). Such works always agree in finding $M_{*}$ values slightly fainter than $M_{*}^{\text {total }}$ and small deviations from a flat faint-end. 
Table 3. Comparison with other works: field galaxies.

\begin{tabular}{|c|c|c|c|c|c|}
\hline Reference & Catalog & $z_{\lim }$ & $\alpha$ & $M_{*}-5 \log h$ & $\phi\left(10^{-3} h^{3} \mathrm{Mpc}^{-3}\right)$ \\
\hline \multicolumn{6}{|c|}{ Local samples $(z \leq 0.1)$} \\
\hline This paper & $\mathrm{UZC}$ & 0.02 & $-0.95 \pm 0.07$ & $-20.32 \pm 0.10$ & $4.8 \pm 0.5$ \\
\hline Davis \& Huchra (1982) & CfA & $<0.05$ & -0.90 & -20.14 & 3.0 \\
\hline Choloniewski (1986) & CfA & $<0.05$ & $-1.09 \pm 0.07$ & $-20.14 \pm 0.07$ & $3.0 \pm 0.6$ \\
\hline Efstathiou et al. (1988) & CfA & $<0.05$ & -0.96 & -20.10 & 5.8 \\
\hline de Lapparent et al. (1989) & CfA & 0.03 & $-1.10 \pm 0.10$ & $-20.14 \pm 0.10$ & $5.5 \pm 1.4$ \\
\hline Marzke et al. (1994a) & CfA & 0.03 & $-1.00 \pm 0.20$ & $-19.74 \pm 0.30$ & $11.0 \pm 3.0$ \\
\hline Marzke et al. (1994b) & CfA & 0.04 & -1.02 & -19.84 & $5.5 \pm 1.4$ \\
\hline Marzke et al. (1998) & SSRS2 & 0.05 & $-1.08 \pm 0.06$ & $-20.34 \pm 0.06$ & $3.8 \pm 0.6$ \\
\hline Marzke \& da Costa (1997) & SSRS2 & 0.05 & $-1.16 \pm 0.08$ & $-20.39 \pm 0.08$ & $3.0 \pm 0.8$ \\
\hline Ratcliffe et al. (1998) & $\mathrm{D} / \mathrm{UKST}$ & 0.05 & $-1.04 \pm 0.08$ & $-20.62 \pm 0.08$ & $4.7 \pm 0.8$ \\
\hline Marinoni et al. (1999) & NOG & 0.02 & $-1.15 \pm 0.05$ & $-20.65 \pm 0.08$ & $3.9 \pm 0.7$ \\
\hline Loveday et al. (1992) & S-APM & 0.1 & $-0.97 \pm 0.15$ & $-20.44 \pm 0.13$ & $3.8 \pm 0.6$ \\
\hline Lin et al. (1996) & LCRS & 0.1 & $-0.70 \pm 0.03$ & $-20.27 \pm 0.02$ & $5.2 \pm 0.3$ \\
\hline Ellis et al. (1996) & Autofib & $<0.10$ & $-1.09 \pm 0.10$ & $-20.14 \pm 0.30$ & $7.1 \pm 2.2$ \\
\hline \multicolumn{6}{|c|}{ Deep samples $(z>0.1)$} \\
\hline Geller et al. (1997) & $\mathrm{CS}$ & 0.15 & $-1.17 \pm 0.19$ & $-20.37 \pm 0.18$ & $6.8 \pm 1.6$ \\
\hline Ellis et al. (1996) & Autofib & $<0.15$ & $-1.16 \pm 0.05$ & $-20.24 \pm 0.15$ & $6.8 \pm 1.1$ \\
\hline Ellis et al. (1996) & Autofib & $0.15<z<0.35$ & $-1.41 \pm 0.12$ & $-20.59 \pm 0.12$ & $4.1 \pm 0.8$ \\
\hline Ellis et al. (1996) & Autofib & $0.35<z<0.75$ & $-1.45 \pm 0.18$ & $-20.32 \pm 0.27$ & $9.9 \pm 1.1$ \\
\hline Madgwick et al. (2002) & $2 \mathrm{dF}$ & 0.15 & $-1.19 \pm 0.01$ & $-20.73 \pm 0.04$ & $4.4 \pm 0.3$ \\
\hline Norberg et al. (2002) & $2 \mathrm{dF}$ & 0.25 & $-1.18 \pm 0.03$ & $-20.42 \pm 0.11$ & $5.8 \pm 0.6$ \\
\hline Folkes et al. (1999) & $2 \mathrm{dF}$ & 0.2 & $-1.28 \pm 0.05$ & $-20.67 \pm 0.06$ & $4.7 \pm 0.6$ \\
\hline Zucca et al. (1997) & ESP & 0.16 & $-1.22 \pm 0.07$ & $-20.55 \pm 0.08$ & $5.5 \pm 1.1$ \\
\hline Blanton et al. (2001) & SDSS & 0.2 & $-1.15 \pm 0.05$ & $-20.56 \pm 0.04$ & $6.3 \pm 0.8$ \\
\hline Gardner et al. (1997) & K-Survey & 0.3 & $-0.91 \pm 0.10$ & $-20.11 \pm 0.10$ & 4.7 \\
\hline
\end{tabular}

When the different types of field S/I galaxies are analyzed separately, previous works also find the same trends as those obtained in this paper. With the only exception of Efstathiou et al. (1988), the LF of early spirals typically exhibits a declining faint-end ( $\alpha \simeq-0.5$ ) while that of $\mathrm{Sd} / \mathrm{I}$ galaxies typically has a steeply increasing faint-end $(\alpha \simeq-1.8)$. In addition, with the only exception of Marzke et al. (1994a), the $M_{*}$ value of early spirals is generally fainter than that found for $\mathrm{Sd} / \mathrm{I}$ objects.

Other authors have instead analyzed the spectral type dependence of the galaxy luminosity function as derived from the LCRS (Bromley et al. 1998), the 2dFRS (Folkes et al. 1999; de Propis et al. 2002; Madgwick et al. 2002) and the Autofib redshift survey (Heyl et al. 1997). Such works show a more progressive steepening of the logarithmic slope from early to late types than that observed in works analyzing the morphological type dependence. Furthermore, the $M_{*}$ value becomes progressively fainter (instead of brighter) from early to late spectral types. Such a different behavior can be explained (Marinoni et al. 1999) from the fact that galaxy typing based on the visible morphological appearance is not very tightly correlated with galaxy classifications based on spectral schemes.
The latter method is in fact very sensitive to the degree of star formation activity.

\subsection{Luminosity functions in different environments}

\subsubsection{Cluster galaxies}

Most previous works on the overall LF of moderately rich clusters agree with us in finding substantial differences with respect to that measured for field galaxies. Such works concur with $M_{*}$ values brighter by about 1 mag than those of the field galaxy LFs, whereas there is no agreement on whether the LF slope $\alpha$ of cluster galaxies differs significantly from that of the local field. Although some authors (Garilli et al. 1999; Paolillo et al. 2001; Goto et al. 2002) find a nearly flat logarithmic slope, most other works (see Table 5) obtain that the logarithmic slope of cluster galaxies exhibits an increasing behavior $(-1.50 \lesssim \alpha \lesssim-1.22)$.

In the particular case of the Virgo cluster, most authors (Sandage et al. 1985; Ferguson \& Sandage 1988, 1991) found a Schechter $\alpha$ value of about -1.3 , similar to that found in this 
Table 4. Comparison with other works: type-specific LFs.

\begin{tabular}{|c|c|c|}
\hline Reference & $\bar{\alpha}$ & $M_{*}^{\mathrm{typ}}-M_{*}^{\mathrm{tot}}$ \\
\hline \multicolumn{3}{|c|}{ E/S0 galaxies } \\
\hline This paper & $-0.88 \pm 0.13$ & $-0.23 \pm 0.30$ \\
\hline Efstathiou et al. (1988) & -0.88 & -0.07 \\
\hline Loveday et al. (1992) & $+0.20 \pm 0.35$ & $-0.21 \pm 0.38$ \\
\hline Marinoni et al. (1999) & $-1.12 \pm 0.13$ & $-0.08 \pm 0.27$ \\
\hline Marzke et al. (1994a) & -0.92 & +0.03 \\
\hline Marzke et al. (1998) & $-0.99 \pm 0.10$ & $+0.02 \pm 0.17$ \\
\hline Tammann et al. (1979) & -0.90 & -0.06 \\
\hline \multicolumn{3}{|c|}{$\mathrm{S} / \mathrm{I}$ galaxies } \\
\hline This paper & $-0.92 \pm 0.10$ & $+0.16 \pm 0.25$ \\
\hline Efstathiou et al. (1988) & -0.96 & +0.07 \\
\hline Loveday et al. (1992) & $-0.80 \pm 0.20$ & $+0.10 \pm 0.29$ \\
\hline Marinoni et al. (1999) & $-1.17 \pm 0.07$ & $+0.04 \pm 0.17$ \\
\hline Tammann et al. (1979) & -1.10 & +0.08 \\
\hline \multicolumn{3}{|c|}{ E galaxies } \\
\hline This paper & $-1.16 \pm 0.20$ & $-1.46 \pm 1.00$ \\
\hline Efstathiou et al. (1988) & -0.92 & -0.39 \\
\hline Marinoni et al. (1999) & $-0.55 \pm 0.22$ & $-0.09 \pm 0.35$ \\
\hline Marzke et al. (1994a) & -0.85 & -0.33 \\
\hline Tammann et al. (1979) & -0.77 & -0.26 \\
\hline \multicolumn{3}{|c|}{ S0 galaxies } \\
\hline This paper & $-0.69 \pm 0.20$ & $+0.18 \pm 0.35$ \\
\hline Efstathiou et al. (1988) & -0.84 & +0.16 \\
\hline Marinoni et al. (1999) & $-1.08 \pm 0.21$ & $+0.46 \pm 0.32$ \\
\hline Marzke et al. (1994a) & -0.94 & +0.16 \\
\hline Tammann et al. (1979) & -0.46 & +0.67 \\
\hline \multicolumn{3}{|c|}{ Early spiral galaxies } \\
\hline This paper & $-0.43 \pm 0.45$ & $+0.63 \pm 0.46$ \\
\hline Efstathiou et al. (1988) & -1.00 & +0.17 \\
\hline Marinoni et al. (1999) & $-0.78 \pm 0.11$ & $+0.07 \pm 0.21$ \\
\hline Marzke et al. (1994a) & -0.58 & +0.18 \\
\hline Tammann et al. (1979) & -0.47 & +0.62 \\
\hline \multicolumn{3}{|c|}{ Late $\mathrm{S} / \mathrm{I}$ galaxies } \\
\hline This paper & $-2.15 \pm 0.13$ & $-0.35 \pm 0.42$ \\
\hline Efstathiou et al. (1988) & -1.20 & -0.13 \\
\hline Marinoni et al. (1999) & $-2.27 \pm 0.32$ & $-0.11 \pm 0.77$ \\
\hline Marzke et al. (1994a) & -1.87 & +0.11 \\
\hline Marzke et al. (1998) & $-1.74 \pm 0.25$ & $-0.29 \pm 0.54$ \\
\hline
\end{tabular}

paper. This logarithmic slope is even much more steeply increasing $(\alpha<-2)$ when very faint galaxies are considered (Trentham \& Hodgkin 2002) due to the presence of new galaxy populations (such as dwarf galaxies).

In contrast with the Virgo cluster, the Ursa Major cluster has received relatively little attention in the specialized literature for two main reasons (Tully et al. 1996). Firstly, it is a poorly defined cluster which lacks concentration to any core. Secondly, the Ursa Major cluster lies in a particularly confusing part of the sky because it is in the plane of the Local Supercluster at the junction of filamentary structures. Its luminosity function has been recently analyzed by Trentham et al. (2001), who found a galaxy distribution quite different from
Table 5. Comparison with other works: group galaxies.

\begin{tabular}{lll}
\hline \hline \multicolumn{1}{c}{ Reference } & \multicolumn{1}{c}{$M_{*}$} & \multicolumn{1}{c}{$\alpha$} \\
\hline & Isolated galaxies \\
This paper & $-20.57 \pm 0.29$ & $-1.21 \pm 0.15$ \\
Marinoni et al. (1999) & $-20.64 \pm 0.12$ & $-1.18 \pm 0.09$ \\
Ramella et al. (1999) & $-20.46 \pm 0.10$ & $-1.21 \pm 0.10$ \\
& Galaxy groups \\
This paper & $-20.34 \pm 0.27$ & $-0.78 \pm 0.19$ \\
Ferguson et al. (1991) & -20.55 & -1.16 \\
Ribeiro et al. (1994) & $-19.90 \pm 0.16$ & $-0.82 \pm 0.09$ \\
Zepf et al. (1997) & $-20.14 \pm 0.20$ & $-1.00 \pm 0.20$ \\
Muriel et al. (1998) & $-20.54 \pm 0.20$ & $-1.00 \pm 0.20$ \\
Zabludoff et al. (2000) & $-21.24 \pm 0.40$ & $-1.30 \pm 0.10$ \\
\multicolumn{1}{c}{ Very poor galaxy clusters (Ursa Major) } \\
This paper & $-20.53 \pm 1.10$ & $-0.81 \pm 0.45$ \\
Trentham et al. (2001) & -20.45 & $-1.01 \pm 0.2$ \\
\multicolumn{1}{c}{ Moderately rich galaxy clusters } \\
This paper & $-21.01 \pm 0.32$ & $-1.38 \pm 0.11$ \\
Sandage et al. (1985) & -20.53 & -1.30 \\
Ferguson et al. (1991) & -20.61 & -1.34 \\
Schechter (1976) & $-20.84 \pm 0.50$ & $-1.24 \pm 0.05$ \\
Dressler (1978) & $-20.64 \pm 0.50$ & -1.25 \\
Lugger (1986) & $-20.79 \pm 0.66$ & $-1.24 \pm 0.22$ \\
Colless (1989) & $-21.04 \pm 0.07$ & -1.25 \\
Lumsden et al. (1997) & $-21.10 \pm 0.02$ & $-1.22 \pm 0.04$ \\
Valotto et al. (1997) & $-20.94 \pm 0.10$ & $-1.40 \pm 0.10$ \\
Rauzy et al. (1998) & $-20.85 \pm 0.21$ & $-1.50 \pm 0.11$ \\
Garilli et al. (1999) & $-21.24 \pm 0.10$ & $-0.94 \pm 0.07$ \\
Paolillo et al. (2001) & $-21.16 \pm 0.15$ & $-1.07 \pm 0.08$ \\
Goto et al. (2002) & $-21.55 \pm 0.11$ & $-1.00 \pm 0.06$ \\
de Propis et al. (2002) & $-21.01 \pm 0.07$ & $-1.28 \pm 0.03$ \\
\hline & & \\
\hline & & \\
\hline
\end{tabular}

† Small groups; ${ }^{\ddagger}$ Virgo cluster.

that seen in the Virgo cluster. In particular, the Ursa Major LF exhibited a flat logarithmic slope $\alpha=-1.01$ for galaxies brighter than $M_{R}=-18$. Even when very faint galaxies were considered (down to $M_{R}=-11$ ) the logarithmic slope obtained by Trentham et al. (2001) for the Ursa Major cluster remained nearly flat: $\alpha=-1.1$. All these values are compatible with our results. Nevertheless, the Schechter parameters obtained in the present paper for the Ursa Major cluster have large uncertainties which mainly arise from the friend-of-friend algorithm.

\subsection{Group galaxies}

Past determinations of the LF for galaxies in small groups differ widely. Some works (e.g., Ferguson \& Sandage 1991; Muriel et al. 1998) on various nearby groups of galaxies agree in finding luminosity functions (for $M_{B}$ values brighter than -16) which are similar to the flat LF generally observed for field samples. The analysis of compact groups (Ribeiro et al. 1994; Zepf et al. 1997) also leads to a flat or a moderately 
decreasing faint-end of the LF of group galaxies. In the opposite, Zabludoff \& Mulchaey (2000) found a steeply increasing logarithmic slope for the LF of group galaxies. We find instead that both small and large groups have LFs with a moderately decreasing logarithmic slope (see Table 2) similar to that found by Ribeiro et al. (1994). Such a discrepancy in the results obtained from different authors is probably due to uncertainties in determining the membership of galaxies to small groups. As a matter of fact, bound members are difficult to be distinguished from chance superpositions of galaxies along the line-of-sight. Consequently, since the number of members per galaxy group is usually small, the incorrect inclusion or exclusion from a group of some few galaxies could have a considerable effect on the resulting LF.

Interestingly, within non-isolated galaxies, the subsample corresponding to moderately rich clusters has a LF not very different from that obtained for isolated galaxies.

\subsection{Isolated galaxies}

Among recent works in which environmental effects on the LF are searched for through a specific comparison between group and field galaxy LFs, an approach similar to ours was followed by Ramella et al. (1999), Bromley et al. (1998) and Marinoni et al. (1999), who divided their respective ESP, LCRS and NOG populations into isolated galaxies and galaxy members of groups or clusters, identified by means of the friendsof-friends algorithm. All these works agree with us in finding Schechter parameters which are significantly different from those obtained for non-isolated galaxies (see Fig. 7).

\section{Conclusions}

In this paper, we have computed the luminosity function of bright nearby galaxies in the Updated Zwicky Catalog and analyzed its dependence on both morphology and environment.

We find a clear morphological dependence of the galaxy LF (see Fig. 3). Different types of galaxies have in fact LFs which significantly differ in their shapes. In the particular case of early and late $\mathrm{S} / \mathrm{I}$ galaxies, the shape variation is mainly due to differences in the logarithmic slope, $\alpha$, of their LFs. Early spirals have LFs with a decreasing faint-end ( $\alpha \simeq-0.43$ ), whereas late $\mathrm{S} / \mathrm{I}$ galaxies $(\mathrm{Sd}+\mathrm{I})$ have LFs with a steeply increasing faint-end $(\alpha \simeq-2.15)$. Intermediate spiral types $(\mathrm{Sb}+\mathrm{Sbc}$ and $\mathrm{Sc}+\mathrm{Scd})$ have instead $\alpha$ values which are compatible, within the uncertainties, with a flat faint-end. These intermediate spiral types essentially differ in the $M_{*}$ value, which is brighter for $\mathrm{Sb}+\mathrm{Sbc}$ galaxies than for Scs. The LFs of elliptical and lenticular galaxies also differ essentially in their $M_{*}$ value, which is brighter for E galaxies.

We have also shown that the specific morphological type is not the only parameter which determines the shape of the galaxy LF. Environmental effects are also important and, therefore, the type-specific LFs have not universal shapes.

In order to analyze the environmental dependence of the LF of galaxies, we have measured the type-specific LF of galaxies in different environments, from isolated to (moderately) rich clusters. From this approach, we find significant differences (see Fig. 7) in the LFs of galaxies with different environments. The overall LF already distinguishes two subsets of environments: On one hand, galaxy groups and poor clusters and, on the other hand, isolated galaxies and moderately rich clusters. The environments included in these two subsets can be in turn distinguished by their type-specific LFs. In particular, the environments included in the former subset (poor clusters, small groups and large groups) have significantly different LFs of $\mathrm{E}+\mathrm{S} 0$ galaxies, whereas the environments included in the latter subset (isolated galaxies and moderately rich clusters) have significantly different LFs of S+I galaxies.

The environmental dependence of the galaxy LF has also been analyzed by using a complementary approach: we have proposed and applied a robust test which examines the hypothesis of universal LFTs. From this approach, we conclude that the observed difference between the overall LF of field and cluster galaxies cannot be explained as a composition, taking into account the relative abundance of Hubble types, of typedependent LFs with universal shapes. Environmental effects also play a crucial role in the shape of the specific LF for each Hubble type. Nevertheless, this dependence appears to be weak enough to allow for the definition of nearly universal luminosity functions, $\Phi(\rho, T)$ with coarse-grained bins on both environment $\rho$, and Hubble type $T$.

Our results clarify the apparent discrepancy between the results previously found by other authors. As a matter of fact, we note that most works claiming the universality of the type-specific LFs have compared clusters with a similar or higher richness than Virgo (Ferguson \& Sandage 1988; Jerjen \& Tamman 1997; Andreon 1998; Trentham 1998), whereas most works claiming an environmental dependence of the typespecific LFs (e.g., Bromley et al. 1998; Ramella et al. 1999; Marinoni et al. 1999) have essentially distinguished two samples: a low-density sample with isolated galaxies only, and a high-density sample constituted by galaxies within a friend-offriend structure (groups or cluster). We note that both results are not incompatible because they do not cover the same range of environments. The results presented in this paper cover from isolated galaxies to moderately rich clusters and imply that LFTs are not universal, in agreement with the former authors. Although we have not analyzed very rich clusters, the results obtained by the latter authors seem to imply almost universal LFTs. We then expect that our results for the Virgo cluster can be extended to clusters much richer than those existing in our sample, at least for the bright range $(M<-17.7)$ of the LF. For very faint magnitudes $(M>>-17.7)$ new galaxy populations arise, as dwarf galaxies, and differences between the LF of moderately and very rich clusters could be possible (Lopez-Cruz et al. 1997; Valotto et al. 1997).

\section{Appendix A: Equivalence of the classical and Choloniewski methods for a complete sample}

The method proposed by Choloniewski (1987) considers a sample of $N_{\mathrm{g}}$ galaxies where data are ordered so that

$M_{k} \leq M_{k+1}$ 
This method starts by counting, for each galaxy $k$, the number $C_{k}$ of sampled galaxies verifying:

$M_{\min } \leq M<M_{k}, \quad$ and $\quad \mu_{\min } \leq \mu \leq m_{\text {lim }}-M_{k}$

where $m_{\lim }$ is the limiting apparent magnitude of the sample and $\mu=25+5 \log r$ is the distance modulus (with the distance $r$ expressed in $\mathrm{Mpc}$ ).

In the case of samples which are complete both in apparent and absolute magnitude, the maximum values of $M$ and $\mu$ satisfy: $M_{\max }+\mu_{\max }=m_{\text {lim. }}$. Consequently, all the sampled galaxies verify the second condition in Eq. (14) and the $C_{k}$ value reduces to the number of galaxies with an absolute magnitude smaller than $M_{k}$. Since the data are ordered according to Eq. (13), one finds that

$C_{k}=k-1$.

The following step in the Choloniewski (1987) method consists of computing the luminosity coefficients, $\psi_{k}$, by using the recursion relation:

$\psi_{k}=\psi_{k-1} \frac{C_{k-1}+1}{C_{k}} \quad\left(k=1, \ldots, N_{\mathrm{g}}\right)$

with $\psi_{1}=1$.

By introducing Eq. (15) into Eq. (16), we obtain that all the $\psi_{k}$ coefficients in a complete sample reduce to:

$\psi_{k}=\psi_{1}=1 \quad\left(k=1, \ldots, N_{\mathrm{g}}\right)$.

Once obtained the $\psi_{k}$ values, the following step in the the Choloniewski (1987) method consists of computing the density coefficients, $d_{k}$, by means of

$1=d_{k} \sum_{j}^{M_{j}+\mu_{k} \leq m_{\lim }} \psi_{j}$

which, in the particular case considered here (where $M_{\max }+$ $\left.\mu_{\max }=m_{\text {lim }}\right)$, reduces to

$d_{k}=1 / N_{\mathrm{g}} \quad\left(k=1, \ldots, N_{\mathrm{g}}\right)$.

Finally, the smoothed value of the luminosity function is given by

$\Phi(M)=\frac{1}{V_{\mathrm{t}} \Delta M} \sum_{k}^{M_{k} \in[M-\Delta M, M+\Delta M]} \psi_{i} \sum_{j}^{N_{\mathrm{g}}} d_{j}$

where $V_{\mathrm{t}}$ is the sampled volume.

Taking into account Eqs. (17) and (19):

$\Phi(M)=\frac{N(M-\Delta M, M+\Delta M)}{V_{\mathrm{t}} \Delta M}$

where $N(M-\Delta M, M+\Delta M)$ is the number of sampled galaxies with $M \in[M-\Delta M, M+\Delta M]$.

On the other hand, in the so-called classical method, the luminosity function is directly estimated from:

$\Phi(M)=\frac{N(M-\Delta M, M+\Delta M)}{V(M) \Delta M}$

where the volume $V(M)$ is determined by the maximum distance an object of absolute magnitude $M$ can have and still be in the sample. In the particular case of volume-limited samples which are complete both in apparent and absolute magnitudes, $V(M)=V_{\mathrm{t}}$ and Eq. (22) reduces to Eq. (21). Consequently, in this case, the classical and $\mathrm{C}$ methods become equivalent.

\section{References}

Andreon, S. 1998, A\&A, 336, 98

Andreon, S., Davoust, E., \& Heim, T. 1997, A\&A, 323, 337

Binggeli, B., Tammann, G. A., \& Sandage, A. 1987, AJ, 94, 251

Binggeli, B., Sandage, A., \& Tammann, G. A. 1988, ARA\&A, 26, 509

Blanton, M. R., Dalcanton, J., Eisenstein, D., et al. 2001, AJ, 121, 2358

Bromley, B. C., Press, W. H., Lin, H., \& Kirshner, R. P. 1998, ApJ, 505,25

Colless, M. M. 1989, MNRAS, 237, 799

Cuesta-Bolao, M. J., \& Serna, A. 2003, in preparation

Choloniewski, J. 1986, MNRAS, 223, 1

Choloniewski, J. 1987, MNRAS, 226, 273

da Costa, L. N., Geller, M. J., Pellegrini, P. S., et al. 1994, ApJ, 424, L1

Davis, M., \& Huchra, J. P. 1982, ApJ, 254, 437

de Lapparent, V., Geller, M. J., \& Huchra, J. P. 1989, ApJ, 343, 1

de Propis, R., Colless, M., Driver, S., et al. 2002, preprint [astro-ph/0212562]

Dressler, A. 1978, ApJ, 223, 765

Dressler, A. 1980, ApJ, 236, 351

Efstathiou, G., Ellis, R. S., \& Peterson, B. A. 1988, MNRAS, 232, 431

Ellis, R. S., Colless, M., Broadhurst, T., Heyl, J., \& Glazebrook, K. 1996, MNRAS, 280, 235

Falco, E. E., Kurtz, M. J., Geller M. J., et al. 1999, PASP, 111, 438

Felten, J. E. 1977, AJ, 82, 861

Ferguson, H. C., \& Sandage, A. 1988, AJ, 96, 1520

Ferguson, H. C., \& Sandage, A. 1991, AJ, 101, 765

Folkes, S., Ronen, S., Price, I., et al. 1999, MNRAS, 308, 459

Gardner, J. P., Sharples, R. M., Frenk, C. S., \& Carrasco, B. E. 1997, ApJ, 480, L99

Garilli, B. M., Maccagni, D., \& Andreon, S. 1999, A\&A, 342, 408

Geller, M. J., Kurtz, M. J., Wegner, G., et al. 1997, AJ, 114, 2205

Goto, T., Okamura, S., McKay, T., et al. 2002, PASJ, 54, 515

Heyl, J., Colless, M., Ellis, R. S., \& Broadhurst, T. 1997, MNRAS, 285,613

Huchra, J. P., \& Geller, M. J. 1982, ApJ, 257, 423

Huchra, J. P., Geller, M. J., Clemens, C. M., Tokarz, S. P., \& Michel, A. 1996, VizieR Online Data Catalog, 7193

Huchra, J., \& Sargent, W. L. W. 1973, ApJ, 186, 433

Jerjen, H., \& Tamman, G. 1997, A\&A, 321, 713

Lin, H., Kirshner, R. P., Shectman, S. A., et al. 1996, ApJ, 464, 60

Lopez-Cruz, O., Yee, H. K. C., Brown, J. P., Jones, C., \& Forman, W. 1997, ApJ, 475, L97

Loveday, J., Peterson, B. A., Efstathiou, G., \& Maddox, S. J. 1992, ApJ, 390, 338

Lugger, P. M. 1986, ApJ, 303, 535

Lynden-Bell, D. 1971, MNRAS, 155, 95

Lumsden, S. L., Collins, C. A., Nichol, R. C., Eke, V. R., \& Guzzo, L. 1997, MNRAS, 290, 119

Madgwick, D. S., Lahav, O., Baldry, I. K., et al. 2002, MNRAS, 333, 133

Marinoni, C., Monaco, P., Giuricin, G., \& Costantini, B. 1999, ApJ, 521,50

Marzke, R. O., \& da Costa, L. N. 1997, AJ, 113, 185

Marzke, R. O., da Costa, L. N., Pellegrini, P. S., Willmer, C. N. A., \& Geller, M. J. 1998, ApJ, 503, 617

Marzke, R. O., Geller, M. J., Huchra, J. P., \& Corwin, H. G. 1994a, AJ, 108, 437

Marzke, R. O., Huchra, J. P., \& Geller, M. J. 1994b, ApJ, 428, 43

Materne, J. 1978, A\&A, 63, 401

Mobasher, B., Sharples, R. M., \& Ellis, R. S. 1993, MNRAS, 263, 560 
Muriel, H., Valotto, C. A., \& Lambas, D. G. 1998, ApJ, 506, 540

Nicol, J. F., \& Segal, I. E. 1983, A\&A, 118, 180

Nolthenius, R. 1993, ApJS, 85, 1

Norberg, P., Cole, S., Baugh, C. M., et al. 2002, MNRAS, 336, 907

Paolillo, M., Andreon, S., Longo, G., et al. 2001, A\&A, 367, 59

Pence, W. 1976, ApJ, 203, 39

Ramella, M., Zamorani, G., Zucca, E., et al. 1999, A\&A, 342, 1

Ratcliffe, A., Shanks, T., Parker, Q. A., \& Fong, R. 1998, MNRAS, 293, 197

Rayzy, S., Adami, C., \& Mazure, A. 1998, A\&A, 337, 31

Ribeiro, A. L. B., de Carvalho, R. R., \& Zepf, S. E. 1994, MNRAS, 267, L13

Sáiz, A., Domínguez-Tenreiro, R., \& Serna, A. 2002, Ap\&SS, 281, 309

Sáiz, A., Domínguez-Tenreiro, R., Tissera, P. B., \& Courteau, S. 2001, MNRAS, 325, 119

Sandage, A. 1973, ApJ, 183, 711

Sandage, A., Binggeli, B., \& Tammann, G. A. 1985, AJ, 90, 1759

Schechter, P. 1976, ApJ, 203, 297

Schechter, P. L. 1980, AJ, 85, 801
Schmidt, M. 1968, ApJ, 151, 393

Serna, A., \& Domínguez-Tenreiro, R. 2001, Highlights of Spanish astrophysics II (Dordrecht: Kluwer Acad. Publ.), 9

Serna, A., \& Gerbal, D. 1996, A\&A, 309, 65

Sommer-Larsen, J., \& Dolgov, A. 2001, ApJ, 551, 608

Tammann, G. A., Yahil, A., \& Sandage, A. 1979, ApJ, 234, 775

Trentham, N. 1998, MNRAS, 294, 193

Trentham, N., \& Hodgkin, S. 2002, MNRAS, 333, 423

Trentham, N., Tully, R. B., \& Verheijen, M. A. W. 2001, MNRAS, 325,385

Tully, R. B., Verheijen, M. A., Pierce, M. J., Huang, J.-S., \& Wainscoat, R. J. 1996, AJ, 112, 2471

Valotto, C. A., Nicotra, M. A., Muriel, H., \& Lambas, D. G. 1997, ApJ, 479, 90

Zabludoff, A. I., \& Mulchaey, J. S. 2000, ApJ, 539, 136

Zepf, S. E., de Carvalho, R. R., \& Ribeiro, A. L. B. 1997, ApJ, 488, L11

Zucca, E., Pozzetti, L., \& Zamorani, G. 1994, MNRAS, 269, 953

Zucca, E., Zamorani, G., Vettolani, G., et al. 1997, A\&A, 326, 477 